\title{
Formation of Bose-Einstein magnon condensate via dipolar and exchange thermalization channels
}

\author{
D.A. Bozhko ${ }^{1,2}$, P. Clausen ${ }^{1}$, A.V. Chumak $^{1}$, Yu.V. Kobljanskyj ${ }^{3}$, \\ B. Hillebrands ${ }^{1}$, and A.A. Serga ${ }^{1}$ \\ ${ }^{1}$ Fachbereich Physik and Landesforschungszentrum OPTIMAS, \\ Technische Universität Kaiserslautern, Kaiserslautern 67663, Germany \\ ${ }^{2}$ Graduate School Materials Science in Mainz, 47 Gottlieb-Daimler-Straße, Kaiserslautern 67663, Germany \\ E-mail: bozhko@physik.uni-kl.de \\ ${ }^{3}$ Faculty of Radiophysics, Electronics and Computer Systems, Taras Shevchenko National University of Kyiv \\ Kyiv 01601, Ukraine
}

Received April 17, 2015, published online August 25, 2015

\begin{abstract}
PACS: $05.30 . J p \quad$ Boson systems;
75.30.Ds Spin waves;

75.70.-i Magnetic properties of thin films, surfaces, and interfaces.
\end{abstract}

Thermalization of a parametrically driven magnon gas leading to the formation of a Bose-Einstein condensate at the bottom of a spin-wave spectrum was studied by time- and wavevector-resolved Brillouin light scattering spectroscopy. Two distinct channels of the thermalization process related on dipolar and exchange parts of a magnon gas spectrum are clearly determined. It has been found that the magnon population in these thermalization channels strongly depends on applied microwave pumping power. The observed magnon redistribution between the channels is caused by the downward frequency shift of the magnon gas spectrum due to the decrease of the saturation magnetization in the course of injection of parametrically pumped magnons.

Keywords: magnon, thermalization, Bose-Einstein condensate.

In ferromagnetic materials, atoms having unpaired electrons act as individual magnets. Since these atomic magnets orient in the same direction due to the exchange interaction, a macroscopic magnetic moment $\mathbf{M}$ appears. In the presence of an external bias magnetic field $\mathbf{H}_{0}$ this magnetic moment tends to be oriented along the field direction. When the equilibrium position of the magnetization is disturbed, $\mathbf{M}$ starts to precess around the equilibrium position. Such precession is described by the classical LandauLifshitz equation of magnetization dynamics [1]:

$$
\frac{d \mathbf{M}}{d t}=-\gamma \mathbf{M} \times \mathbf{H}_{\mathrm{eff}}-\frac{\gamma \lambda}{M^{2}} \mathbf{M} \times\left(\mathbf{M} \times \mathbf{H}_{\mathrm{eff}}\right),
$$

where $\gamma$ is the gyromagnetic ratio and $\lambda$ is a dissipation parameter. An effective internal magnetic field $\mathbf{H}_{\text {eff }}$ includes various terms:

$$
\mathbf{H}_{\mathrm{eff}}=\mathbf{H}_{0}+\mathbf{h}(t)+\int_{V} \tilde{\mathrm{G}}\left(\mathbf{r}, \mathbf{r}^{\prime}\right) \cdot \mathbf{M}\left(\mathbf{r}^{\prime}\right) d r^{\prime 3}+\frac{\eta}{\gamma M_{s}} \nabla^{2} \mathbf{M}+\ldots
$$

where $\tilde{G}\left(\mathbf{r}, \mathbf{r}^{\prime}\right)$ is a tensor Green's function, $\eta$ is a spin stiffness constant, and $M_{S}$ is a saturation magnetization at a given temperature. The first and the second terms in Eq. (2) represent external static and dynamic magnetic fields, respectively. The third term describes an effective dipole-dipole interaction field and the fourth term represents an effective field of the exchange interaction. Equation (2) can also contain the effective fields $\mathbf{H}_{\text {mel }}=$ $=-\delta U_{\mathrm{mel}} / \delta \mathbf{M}, \quad \mathbf{H}_{a}=-\delta U_{a} / \delta \mathbf{M}$, and others, which depend on magneto-elastic interaction energy $U_{\text {mel }}$, energy of magnetic anisotropy $U_{a}$, etc. [2].

In the approximation of a small precession angle, the solution of Eq. (1) with the effective field given by Eq. (2) and proper boundary conditions represents a manifold of spatially non-uniform magnetic excitations - waves of magnetization precession usually called spin waves,whose dispersion characteristics are significantly nonlinear and anisotropic in relation to the direction of the bias magnetic field $\mathbf{H}_{0}$. Figure 1 shows an example of calculation of the first 47 thickness spin-wave modes performed 


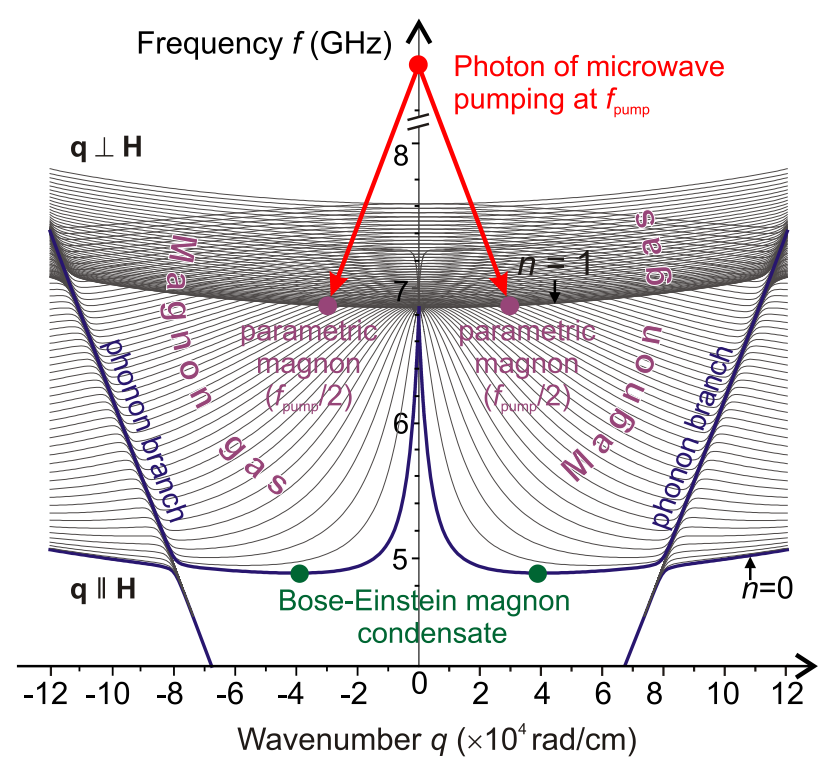

Fig. 1. (Color online) The magnon spectrum calculated in dipoleexchange approximation for the first 47 thickness modes of an inplane magnetized single crystal yttrium iron garnet film of $6.7 \mu \mathrm{m}$ thickness. The external bias field $H_{0}=1735$ Oe, stiffness constant $\eta=9.15 \cdot 10^{-2} \mathrm{~cm}^{2} \cdot \mathrm{s}^{-1}$, saturation magnetization $4 \pi M_{S}=$ $=1750 \mathrm{G}$, the total field of cubic crystallographic and uniaxial out off plane anisotropies $H_{a}=-70$ Oe. Red arrows indicates the magnon injection by parametric pumping. The initially pumped magnon group is marked by two violet dots. Green dots indicates position of the magnon Bose-Einstein condensate.

using the method described in Ref. 3 for two specific propagation directions in an in-plane magnetized ferrimagnetic film of yttrium iron garnet $\left(\mathrm{Y}_{3} \mathrm{Fe}_{5} \mathrm{O}_{12}, \mathrm{YIG}\right)$. Taking into account in the calculation the magneto-elastic field $\mathbf{H}_{\text {mel }}$ leads to the hybridization of all spin-wave modes with the clockwise-polarized phonon branches [4].

As the behavior of the transversal spin-wave branch family ( $\mathbf{q} \perp \mathbf{H}_{0}$ ) is explicitly determined by the exchange interaction their frequencies increase proportionally to $\eta q^{2}$, where $\mathbf{q}$ is an in-plane wavevector. The same exchange-dependent mechanism (see the fourth term in Eq. (2)) is responsible for the increase in the frequency of the high thickness modes ( $n \geq 1)$ having harmonic dynamic magnetization profiles over the film thickness. At the same time, the behavior of the branches from the longitudinal family ( $\mathbf{q} \| \mathbf{H}_{0}$ ) is completely different due to the influence of dynamic demagnetizing fields originating from the dipolar magnetic interaction. For example, in the exchange-free case the frequency of the fundamental spinwave mode $n=0$ (blue curves in Fig. 1) drops monotonically from the ferromagnetic resonance frequency $f_{F M R}=\gamma \sqrt{H_{0}\left(H_{0}+4 \pi M_{z}\right)}$ down to $f_{H}=\gamma H_{0}$ when $|q|$ increases from nearly zero to infinity. Here $M_{Z}$ is a projection of the precessing magnetic moment $\mathbf{M}$ on the direction $\mathbf{z}$ of the static magnetization. However, existence of the exchange interaction leads to the appearance of two global energy minima at finite wavevector values, for the given experimental conditions $q= \pm 4 \cdot 10^{4} \mathrm{rad} / \mathrm{cm}$ as it is shown in Fig. 1.

In terms of the second quantization, a spin-wave ensemble of a magnetic sample can be treated as a gas of weakly interacting quasi-particles called magnons. Each magnon corresponds to the spatially delocalized spin-flip and possesses a magnetic moment of two Bohr magnetons $2 \mu_{B}$. Magnons have an integer spin and, therefore, obey the Bose-Einstein statistics. In the thermal equilibrium the number of magnons depends on the sample temperature and, thus, is not fixed. In such a case the chemical potential of the magnon gas is zero. However, in 1991 it was predicted by Kalafati and Safonov that external injection of magnons can increase the chemical potential up to the bottom of the spin-wave spectrum [5]. These results in the Bose-Einstein magnon condensation: the spontaneous appearance of a coherent state at the global energy minima of the spin-wave spectrum (see Fig. 1).

The most effective way to reach the necessary population of a magnon gas is the parallel parametric pumping, where an alternating magnetic pumping field $\mathbf{h}(t)$ acts along the direction of the bias magnetic field $\mathbf{H}_{0}[7,8]$. This technique is widely used to generate $[9,10]$, amplify [11-13], and restore $[14,15]$ spin-wave signals in macro- and micro-sized magnetic structures. In 2006, this technique was successfully used for the experimental observation of the Bose-Einstein condensate (BEC) of magnons at room temperature [16].

This discovery has attracted the common interest to the physics of the parametrically driven magnon gases, which is now under active theoretical and experimental investigation. Shortly after the first observation, Bugrij and Loktev showed that the condensation of magnons has no restrictions on the temperature of the magnon gas [17]. Later, Rezende [18] presented extended theoretical analyses of the process. Nowadays, the time dependent behavior of the magnon gas in the phase-energy space [19-22], the temperature of the magnon gas [23], and the role of different scattering mechanisms [24] in the process of the thermalization of the injectted magnons are in the focus of attention.

However, the exact mechanism of the thermalization of the parametrically-injected magnons is not clarified yet. In particular, a question about the role of dipolar (dipolar channel of the thermalization) and exchange (exchange channel) magnons in the thermalization process is still not answered. Furthermore, it is expected that specific contributions of the different thermalization channels depend on the spectral position of the initially pumped magnon group, which can vary with change in the pumping power. The mechanism behind this phenomenon is following: with increase in the number $n$ of the excited magnons the magnetization component $M_{Z}$ is reduced [2]:

$$
M_{Z}=M_{S}-g n \mu_{B} .
$$


Here $g$ is the $g$-factor of an electron. Such a reduction may lead to the frequency shift of the magnon spectrum [6] significantly influencing the process of spin-wave parametric excitation and affecting further thermalization dynamics.

Here, we provide experimental insight into the evolution of a magnon gas affected by the four-magnon scattering process in the presence of an external pumping field of different magnitudes. We show that the magnons initially pumped to the low wavenumber region of the perpendicular spin-wave branch (magnon wavevector $\mathbf{q} \perp \mathbf{H}_{0}$ ) scatter into the lowest energy states located at the longitudinal branch (q $\| \mathbf{H}_{0}$ ) passing through both dipolar and exchange parts of the spectrum. With increase in the pumping power the initially pumped magnons occupy the large wavenumber region of the transverse spin-wave branch and transition to the bottom occurs mainly through the exchange region of spectrum. Such a thermalization can be followed by the formation of a magnon BoseEinstein condensate.

The measurements were performed using a lowdamping YIG film by means of a combined microwave and Brillouin light scattering (BLS) setup schematically shown in Fig. 2. The YIG film of $6.7 \mu \mathrm{m}$ thickness with a saturation magnetization $4 \pi M_{S}=1750 \mathrm{G}$ was grown in the (111) crystallographic plane on a gallium gadolinium garnet substrate by liquid phase epitaxy. The YIG-film was in-plane magnetized along the $\langle 110\rangle$ axis. In this case, the crystallographic anisotropy fields can be easily considered in the calculation of the magnon spectrum shown in Fig. 1 [25]. The sample with lateral dimensions of $2 \cdot 10 \mathrm{~mm}^{2}$ was

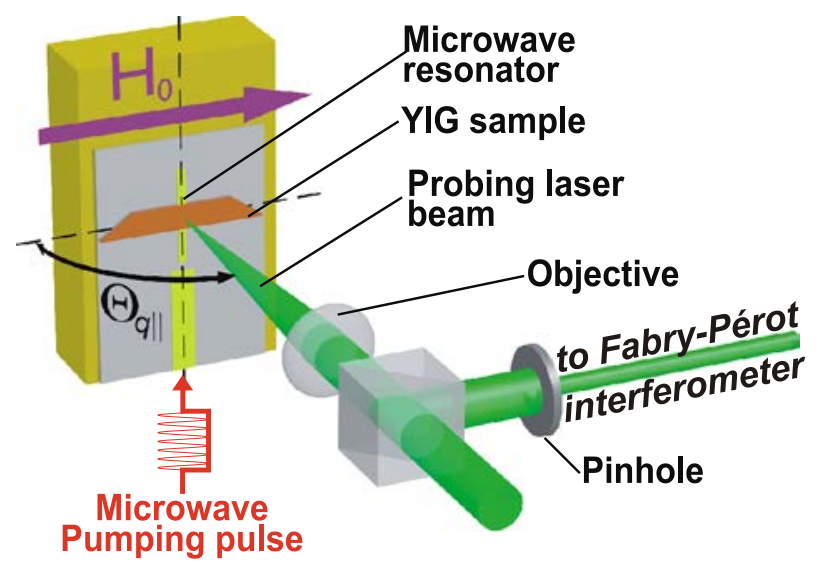

Fig. 2. (Color online) Geometry of the experiment. The setup allows to vary the probing laser beam incidence angle $\Theta_{q \|}$ allowing for wavevector resolution. The YIG sample is placed on top of a microstrip resonator which is fabricated on top of an alumina substrate. Microwave pumping pulses are applied to the microstrip in order to drive the spin-wave system. The probing laser beam is directed through a beam splitter and focused by objective on to the YIG sample. The inelastically scattered light is redirected to the Fabry-Pérot interferometer. An additional pinhole is used to increase the wavenumber resolution. placed on top of a $50 \mu \mathrm{m}$ wide microstrip resonator, which was used to induce the pumping Oersted field.

The resonator was driven by microwave pulses at a carrier frequency of $f_{p}=\omega_{p} / 2 \pi=13.74 \mathrm{GHz}$ with peak powers $P_{p}$ ranging from $1.25 \mathrm{~W}$ to $40 \mathrm{~W}$. The chosen pump pulse duration of 100 ns was sufficiently long to observe the response of the spin-wave system even at the lowest applied pumping powers. A pulse repetition time of $12.5 \mu$ s was chosen to give the system enough time to relax to the ground state and to prevent microwave-heating effects.

The magnon dynamics was analyzed by means of time- and wavevector-resolved BLS spectroscopy [26] with a time resolution of 1 ns. Both the sample and the magnetic system are mounted on a rotating stage in order to be able to change the angle $\Theta_{q \|}$ between the YIG sample and the probing laser beam holding the magnetization conditions constant. By varying this angle, spin waves with different wavevectors parallel to the external magnetic field $\mathbf{H}_{0}$ can be resolved. A three-dimensional pivotal lever utilizing a small xyz-moving stage (not shown in Fig. 2) allows for a precise positioning of the sample. The magnetic system comprises two NdFeB permanent magnets embedded in an iron yoke. The permanent magnets ensure long-term stability of the bias magnetic field in combination with a sufficiently large field strength. Iron shunts are used to tune the magnetic field strength inside the pole gap. For the automated fine control of the bias magnetic field a small electric coil in combination with a Hall sensor is installed. As a result, the magnetic field strength can be adjusted in the range from 1200 to 2100 Oe with an accuracy better than 0.5 Oe. The probing light beam of $532 \mathrm{~nm}$ wavelength and $32 \mathrm{~mW}$ power is generated by a single-mode solid-state laser. The incident light is redirected by a beam-splitter cube and focused by a precise aspherical objective onto the sample. The diameter of the focal point is about $25 \mu \mathrm{m}$. The same objective collects the inelastically scattered light. A pinhole placed behind the objective and the beam-splitter cube assures that only the light coming directly through the middle of the objective can pass to the interferometer. It increases the sensitivity of the optical setup to the small declination of the scattered light, and consequently improves the wavevector resolution. For a proper focusing and spatial stabilization the setup is equipped with a CCD camera and a white light source (not shown in Fig. 2) installed behind the beam-splitter cube and enabling direct observation of the sample. Described setup allowed us to resolve wide longitudinal wavenumber range up to $15 \cdot 10^{4} \mathrm{rad} / \mathrm{cm}$. The wavevector resolution was set to $2 \cdot 10^{3} \mathrm{rad} / \mathrm{cm}$ by a pinhole in the path to the interferometer. In the reported experiment, the frequency resolution of the BLS setup is $100 \mathrm{MHz}$.

In course of the parallel pumping process the photons of the microwave pumping field split into magnon pairs with opposite wavevectors at half of the pumping frequency as 
illustrated in Fig. 1. The strength of the bias field $H_{0}=1735$ Oe has been chosen to allow for a pumping of the magnon pairs slightly above the FMR frequency. In this case, firstly, the parallel pumping achieves its highest efficiency because the magnons are pumped to the transversal spectral branch, which has the lowest threshold of the parametric instability [6]. Secondly, no kinetic instability process, which corresponds to a one-step scattering of the parametric magnons to the bottom of the spin-wave spectrum [27] and which may disturb the formation of the magnon BEC, is allowed due to energy and momentum conservation [28].

Let us address now the formation of the magnon BEC and specifically a question regarding the influence of the injected magnon quantity (i.e., different microwave pumping powers $P_{p}$ ) on the transition of thermalized magnons to the bottom of the spectrum. Figure 3 represents a wavenumber-resolved BLS intensity maps of the time evolution of gaseous magnons with $\mathbf{q} \| \mathbf{H}_{0}$ within the frequency range from $4.7 \mathrm{GHz}$ to $5.7 \mathrm{GHz}$, which includes the bottom of the magnon spectrum. The spectra are normalized to their max- imum. The intensity scale is logarithmic, with low intensities displayed in blue and high displayed in red. Edges of the microwave pulse are marked with vertical white dot lines at 0 and 100 ns. A dark gap, which is visible in Fig. 3(a)-(d) in all four measured spectra at wavevector of $7.9 \cdot 10^{4} \mathrm{rad} / \mathrm{cm}$, corresponds to the area of hybridization between the fundamental spin-wave mode and the clockwise-polarized phonon branch [4]. The formation of the magnon BECs is clearly visible at $4 \cdot 10^{4} \mathrm{rad} / \mathrm{cm}$ in Fig. 3(a)-(c) after switching off the microwave pump pulse. However, during the action of the pump pulse the formation of the magnon BEC is prevented by the extremely high effective temperature of the parametrically driven magnon gas [23]. After the end of the pumping, the magnon temperature rapidly decreases due to the evaporative supercooling process [23] and magnons form the Bose-Einstein condensate. It is worth to note that the magnon BEC formation is not observed in Fig. 3(d), because the microwave pumping power does not exceed the threshold of the magnon Bose-Einstein condensation.

In the case of the highest pumping power $P_{p}=40 \mathrm{~W}$ (see Fig. 3(a)), the first magnons appear at the bottom of the
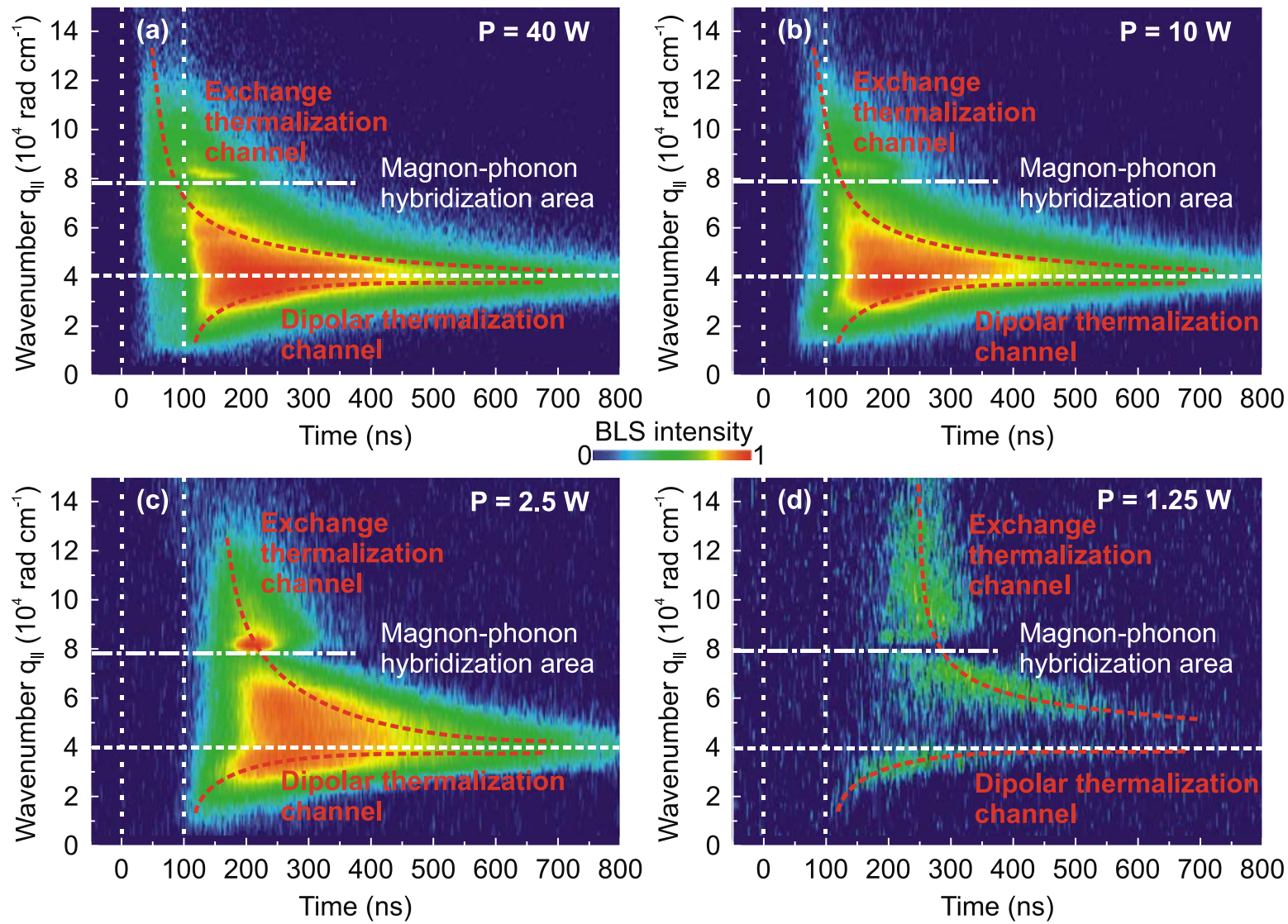

Fig. 3. (Color online) Dependence of the density of thermalized magnons near the bottom of the spin-wave spectrum on the pumping power $P_{p}$, W: 40 (a), 10 (b), 2.5 (c), 1.25 (d). Vertical dotted lines mark the moments when the microwave pumping pulse was switched on and off. Dashed horizontal lines indicate the position of the global energy minima at $|\mathbf{q}|=4 \cdot 10^{4} \mathrm{rad} / \mathrm{cm}$ in the spin-wave spectrum. Red curves in panels (a)-(d) schematically show the time dependent transition paths of dipolar and exchange thermalized magnons to the bottom of the spin-wave spectrum. The formation of the magnon BEC at the global energy minima can be observed in the panels (a)-(c). The gap in the magnon density distribution is visible in all panels around the dashed-dotted lines indicating the magnon-phonon hybridization area (see also Fig. 1). 
spin-wave spectrum already $30 \mathrm{~ns}$ after the pump pulse in the entire wavevector range of (1-15) $10^{4} \mathrm{rad} / \mathrm{cm}$. The short transition time is a consequence of the high rate of magnonscattering processes due to the large population of the primary group of magnons. The four-magnon scattering processes, which are caused by the exchange interaction (see the fourth term in Eq. (2)) constitutes the main mechanism for the magnons gas thermalization. This process conserves the number of magnons in the gas and leads to the redistribution of magnons in a phase space. The center of the density distribution of the initially thermalized magnons lies in the exchange region of the spectrum $7 \cdot 10^{4} \mathrm{rad} / \mathrm{cm}$. In the same time the magnon density in a range of (1-4) $10^{4} \mathrm{rad} / \mathrm{cm}$ is relatively weak (take, please, into account the used logarithmic intensity scale). In the case of the lower microwave pumping power $P_{p}=10 \mathrm{~W}$ shown in Fig. 3(b), the first magnons appear after 50 ns delay time in the dipolar thermalization channel in the wavenumber range of $(1-5) \cdot 10^{4} \mathrm{rad} / \mathrm{cm}$ and only starting from $75 \mathrm{~ns}$ the exchange channel is populated (the corresponding thermalization channels are indicated with red dashed guidelines for the eye in Fig. 3(a)-(d)). In Fig. 3(c) (pumping power $P_{p}=2.5 \mathrm{~W}$ ), the first magnons appear only after switching off the pump pulse with 105 ns delay - the smaller density of the initially injected magnons results in a lower four-magnon scattering rate and causes, thus, the longer termalization time. As previous, the first magnons appears in the dipolar spectral region $\left(\sim 2 \cdot 10^{4} \mathrm{rad} / \mathrm{cm}\right)$. In Fig. 3(d) the pumping power is decreased to $P_{p}=$ $=1.25 \mathrm{~W}$. In this case, the densities of thermalized magnons are very weak but still detectable. The two distinct areas of thermalization, which are separated by the energy minimum at a wavevector of $4 \cdot 10^{4} \mathrm{rad} / \mathrm{cm}$, are well recognizable. The first magnons, which appear in the dipolar dominated region in the wavevector range of $(1-4) \cdot 10^{4} \mathrm{rad} / \mathrm{cm}$, are visible beginning from $175 \mathrm{~ns}$, after the pump pulse is switched off. In the wavevector range (4-15) $10^{4} \mathrm{rad} / \mathrm{cm}$ belonging to the exchange-dominated spectral area, the magnon density rises to a detectable level starting from 175 ns. The observed dynamics can be summarized in the following way. One can admit, that the relative time shift of the dipolar thermalization channel practically does not depend on applied microwave power. In the same time, thermalized magnons appear in the exchange spectral area with a delay that strongly depends on the pumping power. This delay shortens with increasing of the pumped magnons density.

The explanation of observed thermalization behavior is following. As it was already mentioned, the strength of the external magnetic field $H_{0}$ was chosen in such a way that in the case of the highest pump power the parametric magnons were injected into the exchange spectral area just above the FMR frequency. It is necessary to note that in this case the spin-wave spectrum is already shifted to lower frequencies by a huge amount of the injected magnons reducing the magnetization $M_{z}$ in accordance with Eq. (3). For a small quantity of the pumped magnons this shift is less pronounced and the spectral position of the magnon injection is located at or even below the FMR frequency. As a result, at the beginning of the pumping process, the parametric magnons are injected into the long-wavelength dipolar area [29] in all experimental cases presented in Fig. 3. This explains why in Fig. 3(a)-(d) the dipolar thermalization area always gets populated in the same way - as the magnon density increases during the action of the pumping pulse, the spectrum shifts down and the pumping point moves to the exchange area of the spectrum. As a result no more magnons are injected into the dipolar area. Thus, the resulting population of this thermalization channel does not change significantly with the increase in the pumping power from $P_{p}=2.5 \mathrm{~W}$ to $40 \mathrm{~W}$.

Another situation occurs in the exchange thermalization region. With increase of the pumping power, magnon density increases faster, so this region starts to be populated earlier. Moreover, since the magnon number increases during the pumping process, injection points continuously shifts towards the higher wavevectors. This leads to the wide spreading of thermalized magnons in the phase space (see exchange areas in Fig. 3(a)-(c)), and thus enhances efficiency of the magnon gas thermalization. In addition, as it is shown in Ref. 30, at high pumping powers a second group of short-wavelength magnons is excited at a half of the pumping frequency significantly increasing the population of the exchange thermalization channel.

Worth to mention, that the injection of magnons below the FMR frequency and subsequent thermalization has already been investigated experimentally in 2008 by Demidov et al. [20] and theoretically in [24]. The pumping conditions were set to inject magnons far below $(\sim 0.5 \mathrm{GHz})$ the FMR frequency, ensuring the efficient population of the dipolar region of the spectrum. However, an accessible wavevector range in the experiment presented in Ref. 20 was not wide enough to probe the exchange region of the spectrum.

In conclusion, we investigated the thermalization of the parametrically driven magnon gas and subsequent magnon Bose-Einstein formation in a phase space. Our experimental findings suggest two channels of the thermalization process, which pass through dipolar and exchange areas of the spin-wave spectrum. During the pumping process, the increasing magnon density causes shift of the spectrum due to the change in the saturation magnetization. This effect leads to the corresponding shift towards higher wavevectors in the position of parametrically injected magnon pairs. It has been shown, that the dipolar thermalization channel doesn't change its qualitative behavior with increase of the pumping power. In the same time, the exchange channel becomes dominating at high pumping powers.

Financial support by the DFG within the SFB/TR49 and Graduate School Materials Science in Mainz is gratefully acknowledged. 
1. L.D. Landau and E.M. Lifshitz, Phys. Z. Sowjetunion 8, 153 (1935).

2. A.G. Gurevich and G.A. Melkov, Magnetization Oscillations and Waves, CRC Press, New York (1996).

3. B.A. Kalinikos and A.N. Slavin, J. Phys. C 19, 7013 (1986).

4. A. Rückriegel, P. Kopietz, D.A. Bozhko, A.A. Serga, and B. Hillebrands, Phys. Rev. B 89, 184413 (2014).

5. Yu.D. Kalafati and V.L. Safonov, Sov. Phys. JETP 73, 836 (1991).

6. A.A. Serga, C.W. Sandweg, V.I. Vasyuchka, M.B. Jungfleisch, B. Hillebrands, A. Kreisel, P. Kopietz, and M.P. Kostylev, Phys. Rev. B 86, 134403 (2012).

7. E. Schlömann, J.J. Green, and U. Milano, J. Appl. Phys. 31, 386S (1960).

8. S.M. Rezende and F.M. de Aguiar, IEEE Proc. 78, 6 (1990).

9. S.O. Demokritov, A.A. Serga, V.E. Demidov, B. Hillebrands, M.P. Kostylev, and B.A. Kalinikos, Nature 426, 159 (2003).

10. T. Brächer, P. Pirro, B. Obry, B. Leven, A.A. Serga, and B. Hillebrands, Appl. Phys. Lett. 99, 162501 (2011).

11. B.A. Kalinikos and M.P. Kostylev, IEEE Transact. Magnet. 33, 3445 (1994).

12. A.V. Bagada, G.A. Melkov, A.A. Serga, and A.N. Slavin, Phys. Rev. Lett. 79, 2137 (1997).

13. T. Brächer, F. Heussner, P. Pirro, T. Fischer, M. Geilen, B. Heinz, B. Lägel, A.A. Serga, and B. Hillebrands, Appl. Phys. Lett. 105, 232409 (2014).

14. G.A. Melkov, Yu.V. Kobljanskyj, A.A. Serga, A.N. Slavin, and V.S. Tiberkevich, Phys. Rev. Lett. 86, 4918 (2001).

15. A.V. Chumak, V.I. Vasyuchka, A.A. Serga, M.P. Kostylev, V.S. Tiberkevich, and B. Hillebrands, Phys. Rev. Lett. 108, 257207 (2012).

16. S.O. Demokritov, V.E. Demidov, O. Dzyapko, G.A. Melkov, A.A. Serga, B. Hillebrands, and A.N. Slavin, Nature 443, 430 (2006).

17. A.I. Bugrij and V.M. Loktev, Fiz. Nizk. Temp. 33, 51 (2007) [Low Temp. Phys. 33, 37 (2007)].
18. S.M. Rezende, Phys. Rev. B 79, 174411 (2009).

19. V.E. Demidov, O. Dzyapko, S.O. Demokritov, G.A. Melkov, and A.N. Slavin, Phys. Rev. Lett. 99, 037205 (2007).

20. V.E. Demidov, O. Dzyapko, M. Buchmeier, T. Stockhoff, G. Schmitz, G.A. Melkov, and S.O. Demokritov, Phys. Rev. Lett. 101, 257201 (2008).

21. V. Demidov, O. Dzyapko, S. Demokritov, G. Melkov, and A. Slavin, Phys. Rev. Lett. 100, 047205 (2008).

22. A. Chumak, G. Melkov, V. Demidov, O. Dzyapko, V. Safonov, and S. Demokritov, Phys. Rev. Lett. 102, 187205 (2009).

23. A.A. Serga, V.S. Tiberkevich, C.W. Sandweg, V.I. Vasyuchka, D.A. Bozhko, A.V. Chumak, T. Neumann, B. Obry, G.A. Melkov, A.N. Slavin, and B. Hillebrands, Nat. Commun. 5, 4452 (2014).

24. J. Hick, T. Kloss, and P. Kopietz, Phys. Rev. B 86, 184417 (2012).

25. V.B. Bobkov, I.V. Zavislyak, and V.F. Romanyuk, J. Commun. Technol. Electron. 48, 196 (2003).

26. C.W. Sandweg, M.B. Jungfleisch, V.I. Vasyucka, A.A. Serga, P. Clausen, H. Schultheiss, B. Hillebrands, A. Kreisel, and P. Kopietz, Rev. Sci. Instrum. 81, 073902 (2010).

27. G.A. Melkov, V.L. Safonov, A.Y. Taranenko, and S.V. Sholom, J. Magn. Magn. Mater. 132, 180 (1994).

28. G.A. Melkov and S.V Sholom, Sov. Phys. JETP 72, 341 (1991).

29. T. Neumann, A.A. Serga, V.I. Vasyuchka, and B. Hillebrands, Appl. Phys. Lett. 94, 192502 (2009).

30. P. Clausen, D.A. Bozhko, V.I. Vasyuchka, G.A. Melkov, B. Hillebrands, and A.A. Serga, Phys. Rev. B 91, 220402(R) (2015). 